\title{
Factores predisponentes a la parvovirosis canina registrados en un hospital de Cuautitlan, México
}

\author{
Aponte, F.A. ${ }^{1}$; Gómez, R.V.'; Lopez, Y.M. ${ }^{1,2}$ \\ ${ }^{1}$ Facultad de Ciencias Agrarias y Ambientales, Fundación Universitaria Juan de \\ Castellanos, Tunja (Colombia). ${ }^{2}$ Maestría en Ciencias Veterinarias, Universidad \\ Pedagógica y Tecnológica de Colombia. E-mail: yovana_mili@hotmail.com
}

\begin{abstract}
Resumen
Aponte, F.A.; Gómez, R.V.; Lopez, Y.M.: Factores predisponentes a la parvovirosis canina registrados en un hospital de Cuautitlan, México. Rev. Vet. 31: 1, 42-45, 2020. El parvovirus canino (PVC) se restringió inicialmente a infectar a los perros, pero luego se extendió a otras familias como felidae (gatos y otros felinos), procionidae (mapaches, coatíes) y ursidae (osos). A pesar de que su epidemiología no ha sido descrita completamente, el clima parece tener influencia directa en los rangos de presentación. En este trabajo se compararon factores como edad, raza, género, estado vacunal y variables climáticas, con la aparición de la enfermedad en un hospital veterinario de Cuautitlan (México). Mediante la revisión de 3.862 historias clínicas de los años 2016-2017, se encontró una prevalencia de $1,86 \%$ ( 72 animales) diagnosticados con PVC, siendo los menores de 7 meses los de mayor susceptibilidad, especialmente durante los meses de marzo a mayo, con mayor incidencia en abril. La variable aumento de la temperatura ambiental explicó la mayor presentación de la enfermedad, seguida por el sexo hembra, el mayor número de crías, la joven edad y el menor consumo de alimento.
\end{abstract}

Palabras clave: canino, parvovirosis, clima, edad, sexo, alimentación.

\begin{abstract}
Aponte, F.A.; Gómez, R.V.; Lopez, Y.M.: Factors that predisposed to canine parvovirosis in a hospital of Cuautitlan, Mexico. Rev. Vet. 31: 1, 42-45, 2020. Canine parvovirus (CPV) was initially described in the Canidae family, but later extended to Ursidae, Procionidae, and Felidae families, among others. Its epidemiology has not been fully described. Nevertheless, climate appears to have a direct influence on the severity of the disease symptomatology. Aim of this study was to analyze the influence of different factors such as age, breed, gender, vaccination status, and climatic variables, by means of analyzing cases of this disease recorded at an veterinary hospital from Cuautitlan (México). For this purpose, clinic histories from 2016 until 2017, were analyzed. Prevalence of $1.86 \%$ was determined, being animals under seven months-old those with the highest susceptibility to CPV. In addition, from March to May, the highest number of cases were diagnosed, with peak on April. The environmental temperature explains the higher presentation of cases of parvovirus. Considering these results, it can be concluded that CPV seems to be more frequent during the warmer months, followed by high number of offspring and lower consumption of food.
\end{abstract}

Key words: dog, parvovirosis, climate, age, sex, feeding, vaccination.

\section{INTRODUCCIÓN}

La parvovirosis canina (PVC) fue detectada a finales de la década de 1970 y a partir de ese momento se han descrito diferentes modificaciones del virus cau$\mathrm{sal}^{3}$. En los estudios de distribución mundial se demostró que el virus de la PVC está relacionado con el virus de la panleucopenia felina (PLF), sospechándose que la PVC probablemente se haya originado a partir de la PLF, dado que entre ambos virus se ha detectado recombinación genética ${ }^{3,5}$.
Taxonómicamente el PVC pertenece a la familia parvoviridae, género protoparvoviris ${ }^{5}$, y se denomina PVC-2, ya que fue el segundo parvovirus descrito en cánidos domésticos, existiendo las cepas PVC-2a, PVC- $2 b$ y PVC- $2 c^{16}$.

El PVC-2 ha sido vinculado a la familia canidae, tanto a especies domésticas como silvestres. El PVC-2, y sus cepas, son altamente contagiosas y de rápida presentación ${ }^{1}$. Afecta tanto a animales domésticos como silvestres, lo cual lo convierte en un agente que puede afectar la salud de las mascotas y la conservación en vida silvestre. 
Los perros que se ven principalmente afectados son los cachorros mayores de 6 meses, aunque pueden enfermarse caninos de cualquier edad, especialmente si no están vacunados ${ }^{17}$.

El PVC-2 se puede transmitir de manera horizontal por vía oro-nasal a partir del contacto con heces contaminadas de caninos enfermos, donde es común el desarrollo de la presentación gastroentérica. También ocurre por vía vertical en el útero después del nacimiento, desarrollando una presentación cardíaca, aunque es poco común debido a la presencia de anticuerpos maternales ${ }^{8}$. Además, en ciertos países, el contagio parece estar asociado a cambios climáticos ${ }^{4,7}$.

El diagnóstico sintomatológico es muy controvertido porque los síntomas no son específicos. No obstante, la aparición súbita de vómitos y diarrea con presencia de moco y sangre, pueden ser indicativos de infección por $\mathrm{PVC}^{8}$. En este caso hay que diferenciarla de otros patógenos que pueden causar gastroenteritis aguda. El diagnóstico definitivo se basa en el aislamiento del PVC en las heces o en el contenido intestinal ${ }^{11}$.

Con tal perspectiva, en este artículo se pretende analizar y relacionar los factores edad, raza, género, estado vacunal y variables climáticas con la presentación de PVC en un hospital veterinario de la ciudad de Cuautitlán, México.

\section{MATERIAL Y MÉTODOS}

Se efectuó la revisión de 3862 historias clínicas existentes en el Hospital Veterinario de Cuautitlán (México) durante 2016 y 2017. Se recolectó información de los perros con diagnóstico de parvovirosis, registrando como variables la raza, el género y la edad. Además, se obtuvieron datos ambientales como temperatura (máxima, mínima y promedio), así como datos pluviométricos obtenidos del Servicio Meteorológico Nacional de México.

Estadísticamente, la prevalencia de parvovirosis se estableció con intervalos de confianza del 95\%, eliminando la variación irregular. Se revisó el corredor endémico temporal de la enfermedad en los meses de enero a abril, de los dos años estudiados, para la cual se obtuvo la media geométrica.

Las variables climáticas se analizaron por medio de la correlación de Pearson. La prevalencia se valoró utilizando la regresión lineal para vincular la presentación de la enfermedad con cada variable climática descrita en el estudio.

\section{RESULTADOS Y DISCUSIÓN}

De los 3862 caninos examinados en el Hospital, 72 de ellos padecían parvovirosis, lo cual equivale a una prevalencia de $1,86 \%$ (IC 95\% = 1,44-2,29\%). Tal prevalencia es baja en comparación con el 76,6\% hallado en otro estudio realizado en México ${ }^{15}$. De los animales positivos a PVC, el 53,9\% fueron hembras, mientras que el $46,1 \%$ fueron machos. En cuanto a las edades, los animales más jóvenes mostraron alta prevalencia de la enfermedad con relación a los mayores a siete meses de edad (Figura 1).

Se evidenció que el $78.9 \%$ de los animales enfermos no habían sido vacunados, mientras que el $21.1 \%$ de los animales vacunados padecieron la enfermedad. Ello puede haberse debido a que algunos animales no generan inmunidad a la vacuna ${ }^{14}$.

Se encontró que la tendencia en la presentación de los casos fue significativamente negativa $(\mathrm{p}<0,05)$, determinada por la ecuación $\mathrm{Y}=-0.4985 \mathrm{X}+0,3765$ (Figura 2) en contraste con lo hallado en décadas de 1980, 1990 y 2010, en los cuales la tendencia fue positiva ${ }^{4,7}$

En el mes de febrero aumentó la presentación de los casos de la enfermedad, teniendo un descenso abrupto en el mes de marzo, seguido de un elevado pico de casos en abril (Figura 3).

El aumento en la presentación de los casos ocurrió al ir elevándose la temperatura del medio ambiente. Ello coincide con lo reportado por investigadores chi-



Figura 1. Prevalencia de PVC según la edad discriminada en meses.

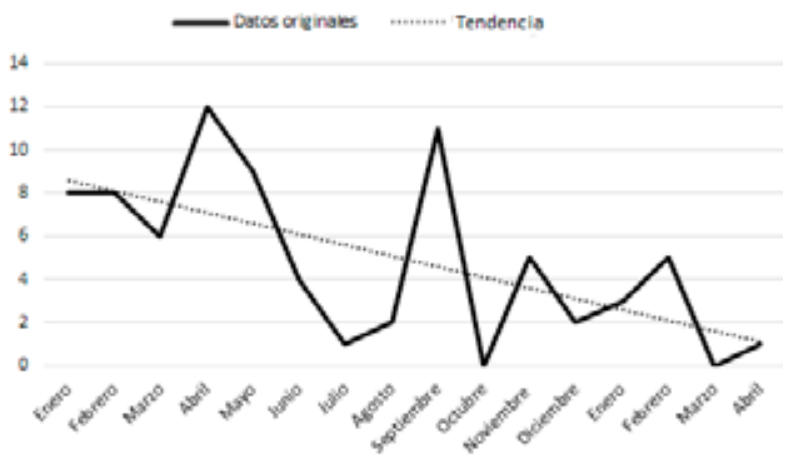

Figura 2. Prevalencia y tendencia de PVC en los años 2016-2017.

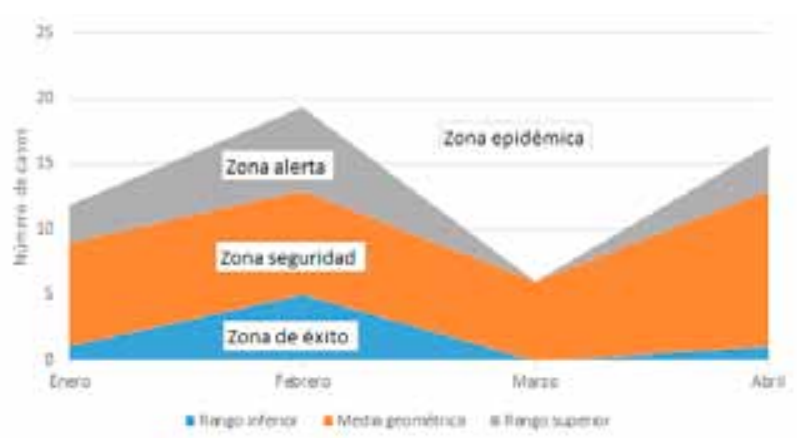

Figura 3. Corredor endémico para casos de PVC en perros de Cuautitlán durante enero a abril de los años 2016 y 2017. 
Tabla 1. Correlación entre las variables climáticas registradas en Cuautitlán.

\begin{tabular}{lccccr}
\hline variables & $\mathrm{T}^{\text {o }}$ máx. & $\mathrm{T}^{\mathrm{o}}$ mín. & $\mathrm{T}^{\mathrm{o}}$ media & viento & pluv. \\
\hline $\mathrm{T}^{\mathbf{o}}$ máx & 1 & & & & \\
$\mathrm{~T}^{\mathbf{o}}$ mín. & 0,9905977 & 1 & & & \\
$\mathrm{~T}^{\text {o }}$ media & 0,9891732 & 0,9942569 & 1 & & \\
viento (v) & 0,7028063 & 0,6992696 & 0,6445189 & 1 & \\
pluv. & 0,8935699 & 0,8982615 & 0,1995090 & 0,3244488 & 1 \\
\hline
\end{tabular}

$\mathrm{T}^{\mathrm{o}}$ : temperatura, máx.: máxima, mín.: mínima, (v): velocidad, pluv.: pluviosidad.

lenos en Valdivia (Chile) ${ }^{7,18}$, quienes hallaron que la parvovirosis se presenta con mayor número de casos en el verano y con un pico en agosto-septiembre.

Este último hallazgo concuerda con el pico encontrado en el mes de septiembre en el presente estudio. De igual forma, en Brasil se halló que en los meses más calurosos del año se eleva el número de casos con PVC, estudio que resultó estadísticamente significativo ${ }^{4}$.

En algunos casos, tal comportamiento estacional se atribuye a la baja ingesta de alimentos en dichos meses y -por lo tanto- a la aparición de una mayor predisposición a sufrir problemas infecciosos, además de ser el momento de mayor número de partos y por lo tanto elevada cantidad de cachorros $2,9,10$.

En estudios realizados en Chile se reportó que el síndrome gastroentérico se presenta principalmente en las estaciones de primavera y otoño, pero tales enfermedades parecen deberse a infecciones bacterianas y virales provocadas por agentes diferentes al PVC por no registrar predominancia estacional ${ }^{12,13}$.

Por otro lado, las variables climáticas mostraron una alta correlación entre ellas, excepto en la velocidad del viento con la pluviosidad (Tabla 1). La temperatura promedio explicó en un $21,10 \%$ la presentación de la enfermedad (Tabla 2), siendo la variable climática que mejor resultado obtuvo en la regresión. La pluviosidad solo explicó el $8.3 \%$ de la prevalencia hallada en el hospital. Estos datos son acordes a otros estudios que demostraron la resistencia del PVC a los cambios de temperatura ambiental ${ }^{6}$.

En conclusión, surge que se halló una tendencia negativa en la prevalencia de PVC en los perros del hospital veterinario de Cuautitlán, México, entre enero de 2016 y abril de 2017. La prevalencia estuvo asociada a los meses del año más calurosos y se presentó principalmente en animales menores de 7 meses de edad. La prevalencia hallada $(1,86 \%)$ es una de las más bajas entre las reportadas para América Latina.

Se recomienda reiterar la investigación ampliando la muestra y anexando un estudio espacial mediante sistemas de información geográfica, para evidenciar los lugares de Cuautitlán que revelen mayor autocorrelación de parvovirus canino.

Agradecimientos. Al Hospital de Enseñanza en Medicina y Cirugía de Pequeñas Especies de la Uni-
Tabla 2. Regresión lineal de variables climáticas para PVC.

\begin{tabular}{lcrc}
\hline variables & $\mathrm{R}$ & \multicolumn{1}{c}{$\mathrm{R}^{2}$} & $\mathrm{CDR}$ \\
\hline $\mathrm{T}^{\mathbf{o}}$ promedio & 0,523 & 0,221 & 0,273 \\
$\mathrm{~T}^{\mathrm{o}}$ máxima & 0,020 & $-0,071$ & 0,001 \\
$\mathrm{~T}^{\mathrm{o}}$ mínima & 0,345 & 0,056 & 0,119 \\
pluviosidad & 0,380 & 0,083 & 0,144 \\
\hline
\end{tabular}

PVC: parvovirosis canina, R: regresión, R: coeficiente de correlación; $\mathrm{R}^{2}$ : coeficiente de determinación; CDR: coeficiente de regresión lineal; $\mathrm{T}^{\mathrm{o}}$ temperatura.

versidad Nacional Autónoma de México, por apoyar la realización de esta investigación.

\section{REFERENCIAS}

1. Candanosa E, Ducoing A, Ruiz RA, Sánchez F. 2007. Diagnóstico del parvovirus canino-2 (pvc-2) por inmunohistoquímica en perros domésticos. Vet Méx 38: 41-53.

2. Carman PS, Povey RC. 1984. The seroprevalence of canine parvovirus-2 in a selected sample of the canine population in Ontario. The Canad Vet J 25: 259-262.

3. Carmichael LE, Schlafer DH, Hashimoto A. 1994. Minute virus of canines (MVC canine parvovirus type-1): pathogenicity for pups and seroprevalence estimate. $J$ Vet Diagn Invest 6: 165-174.

4. Castro TX et al. 2007. Clinical and epidemiological aspects of canine parvovirus enteritis in the State of Rio de Janeiro. Arq Bras Med Vet Zoot 59: 333-339.

5. Cotmore $\mathbf{S}$ et al. 2014. The family parvoviridae. Archives of virology 159: 1239-1247.

6. Decaro N, Buonavoglia C. 2012. Canine parvovirus. A review of epidemiological and diagnostic aspects, with emphasis on type 2c. Vet Microbiol 155: 1-12.

7. Ernst S, Montes S, Huber A. 1987. Prevalencia de parvovirosis clínica en una población canina hospitalaria de Valdivia, Chile. Av Cs Vet 2: 99-104.

8. Ettinger S, Feldman E. 2009. Textbook of veterinary internal medicine, Elsevier Health Sciences, $7^{\circ}$ ed., Saunders, Philadelphia.

9. Houston DM, Ribble CS, Head LL. 1996. Risk factors associated with parvovirus enteritis in dogs: 283 cases (1982-1991). J Am Vet Med Assoc 208: 542-546.

10. Martin R. 1978. Población canina y felina en Chile. Serie Scientia et Tecnica $\mathrm{N}^{\circ}$ 1-1978, Universidad Austral de Chile, Valdivia.

11. Miranda C, Thompson G. 2016. Canine parvovirus in vaccinated dogs: a field study. Vet Rec 16: 397-402.

12. Morales MA, Mora L, Salazar J. 1997. Distemper canino: sobrevida por edad, sexo, raza y estación. Avan Cienc Vet 12: 41-44.

13. Pardo M. 2016. Frecuencia de huevos de nematodos gastrointestinales en heces de perros en el Parque Central Simón Bolívar de Bogotá. Tesis p. título de Méd. Vet. en Univ.La Salle, Colombia. http://repository.lasalle.edu.co/ handle/10185/17543. 
14. Ramsey I, Tennant B. 2012. Manual de enfermedades infecciosas en pequeños animales, Editorial Lexus, Barcelona, p. 381.

15. Ruiz A, Cardona E, Ducang A. 2007. Diagnóstico del parvovirus canino por inmunohistoquímica en perros domésticos. Rev Vet Méx 38: 01.

16. Sosa KA. 2009. Estudio de la diversidad del parvovirus canino tipo 2 (CPV-2). No. 578.822-SOS. https://core. ac.uk/download/pdf/54219842.pdf
17. Tamimi N. 2017. Prevalence of diseases in the canine referred to a private practice in Baghdad in 2015-2016. Kufa $J$ Vet Med Sci 8: 16-23.

18. Vera V. 2012. Análisis espacio-temporal de casos de síndrome de gastroenteritis hemorrágica en caninos, según fichas médicas de una clínica veterinaria de la comuna de Conchalí, período 2000-2008. Tesis para optar al título Médico Veterinario, Escuela de Ciencias Veterinarias, Universidad de Chile, 58 p. 